\title{
Alteração do perfil de atendimento dos hospitais psiquiátricos públicos de Belo Horizonte, Brasil, no contexto da reforma da assistência à saúde mental
}

\author{
Alteration of profile of treatment of the public psychiatric \\ hospitals of Belo Horizonte, Brazil, in the context \\ of mental health care reform
}

\author{
Vívian Andrade Araújo Coelho ${ }^{1}$ \\ Fernando Madalena Volpe ${ }^{2}$ \\ Sabrina Stephanie Lana Diniz ${ }^{1}$ \\ Eliane Mussel da Silva ${ }^{2}$ \\ Cristiane de Freitas Cunha ${ }^{1}$
}

${ }^{1}$ Departamento de Pediatria, Faculdade de Medicina, Universidade Federal de Minas Gerais. Av. Alfredo Balena 190/4 Andar/Sala 275, Santa Efigênia. 30.110-100 Belo Horizonte MG Brasil. vivianaacoelho@gmail.com ${ }^{2}$ Fundação Hospitalar do Estado de Minas Gerais.
Abstract This article seeks to describe the profile of treatment and internment in public psychiatric hospitals in Belo Horizonte, Brazil, from 2002 to 2011. The changes in the characteristics of treatment and the profiles of the patients treated are analyzed in the context of health care reform. It is a study of temporal series with trend analysis by means of linear regression. There was a reduction in the total of patients treated in the period under scrutiny. Inversely, there was an increase in internments with a reduction in length of stay, though no change in readmission rates. Patients from Belo Horizonte prevailed, however a relative increase in demand from the surrounding area was observed. There was a reversal in the prevalence of morbidity switching from psychotic disorders to disorders resulting from the use of alcohol and/or other drugs. The alteration observed in the profile of treatment in public psychiatric hospitals in Belo Horizonte was concomitant with the progressive implementation of community mental health services, which have probably met the demand that was formerly directed to these hospitals. Currently the psychiatric hospital is not the first, much less the only venue for treatment in the mental health network in Minas Gerais.

Key words Psychiatric hospitals, Health care reform, Mental health, Community mental health services
Resumo O objetivo do artigo é descrever os atendimentos e as internações nos hospitais psiquiátricos públicos de Belo Horizonte de 2002 a 2011, explorando mudanças nas características dos atendimentos e no perfil dos pacientes atendidos; no contexto da reforma da assistência em saúde mental. Estudo de séries temporais com análises de tendência por meio de regressões lineares. No período analisado, houve redução no total de atendimentos. Inversamente, constatou-se o aumento das internações com redução do tempo de permanência e manutenção da taxa de reinternação. Os atendimentos a pacientes da capital foram os mais frequentes, entretanto observou-se crescimento relativo da demanda pelos oriundos da Região Metropolitana. Houve inversão na morbidade prevalente de transtornos psicóticos para transtornos decorrentes do uso de álcool e/ou outras drogas. A alteração observada no perfil de atendimento dos hospitais psiquiátricos públicos de Belo Horizonte foi concomitante com a progressiva implantação de serviços comunitários de saúde mental que provavelmente tem suprido uma demanda que antes se dirigia a esses hospitais. Atualmente o hospital psiquiátrico não constitui a primeira e muito menos a única instância de tratamento da rede de saúde mental de Minas Gerais. Palavras-chave Hospitais psiquiátricos, Reforma dos serviços de saúde, Saúde mental, Serviços comunitários de saúde mental 


\section{Introdução}

Os conceitos de saúde e doença mental são polissêmicos e adquirem em cada cultura e em cada época uma caracterização. De acordo com essas concepções, são delineadas maneiras variadas para lidar com o problema, ultrapassando os aspectos técnicos, dentro de um contexto social, político e econômico ${ }^{1}$.

A atuação de Philippe Pinel, no final do século XVIII, é tida como um marco para a assimilação da loucura como doença mental na sociedade ocidental ${ }^{2}$. Criando o conceito de alienação mental, ele definia a loucura como um distúrbio no âmbito das paixões, capaz de produzir desarmonia na mente e na possibilidade objetiva do indivíduo perceber a realidade. Nessa concepção, os conteúdos da mente dependeriam das percepções e das sensações. Assim, para modificar o estado mental seria necessário retirar o alienado de suas percepções habituais ${ }^{3}$. A partir dessa teorização, Pinel concebia o isolamento como necessário para reconduzir o alienado à razão com auxílio da instituição de tratamento ${ }^{4}$.

Apesar de diversas críticas e teorias contrárias às de Pinel não tardarem a aparecer, o hospital se manteve como a instituição preconizada para o tratamento dos doentes mentais até meados do século XX. Essas circunstâncias delinearam para o hospital psiquiátrico uma função médica, terapêutica e uma função social, que se constituía como um ideal de proteção da sociedade perante um grupo que transitava no limite da legalidade e cuja forma de vida não era aceita pela maioria ${ }^{5}$.

Após a Segunda Guerra Mundial, as contestações, diante das denúncias de maus tratos e desumanidade nos hospitais psiquiátricos, vão crescer e impor mudanças ou reformas no asilo e na estrutura do tratamento psiquiátrico ${ }^{6}$. A instituição asilar, que a princípio era uma instituição de cura, passou a ser vista como responsável pelos altos índices de cronificação. Dessa forma, deveria urgentemente ser reformada, pois havia se afastado de sua finalidade ${ }^{7}$. A necessidade de crescimento econômico e de reconstrução social no pós-guerra, a descoberta dos psicotrópicos e a adoção da saúde pública nas instituições psiquiátricas somaram-se como elementos instigantes dos diferentes movimentos de reforma psiquiátrica ocorridos no mundo ${ }^{5}$.

Diversas experiências de transformação do hospital psiquiátrico foram implementadas, principalmente a partir de 1940. Essas podem ser dividas de acordo com seus propósitos: a Comunidade Terapêutica e a Psicoterapia Institucional investiram no princípio de que o fracasso estava na forma de gestão do próprio hospital e que a solução, portanto, seria modificar a instituição; a Psiquiatria de Setor e a Psiquiatria Preventiva, assumiam a comunidade como ponto central para o desenvolvimento do tratamento; já a Antipsiquiatria e Psiquiatria Democrática Italiana, dirigiam os questionamentos à psiquiatria $\mathrm{em} \mathrm{si}$, aos seus saberes e práticas assistenciais 6 .

A reforma psiquiátrica brasileira representa uma proposta de reformulação das formas de lidar com a doença mental no Brasil. Reflexo de movimentos similares ocorridos em outros países e contemporâneo da Reforma Sanitária e da criação do Sistema Único de Saúde (SUS) ${ }^{6}$, esse projeto propôs a reestruturação da assistência à saúde mental no país 8 .

Esse movimento, ainda em curso, constitui um processo político e social complexo iniciado na década de 1970, época em que a institucionalização era o modelo básico de tratamento no país'. A proposta de modificação do modelo de assistência teve como inspiração as reformas ocorridas na Europa e, em especial, o modelo italiano que preconizava a extinção dos hospitais psiquiátricos e sua substituição por serviços substitutivos de atenção comunitária à saúde mental ${ }^{10}$. Assim, não se trataria de aperfeiçoar as instituições tradicionais (ambulatório e hospital de internação), mas sim de inventar novos dispositivos e novas tecnologias de assistência. Dessa forma, o intuito da reforma brasileira é substituir uma psiquiatria centrada no hospital por outra sustentada em dispositivos diversificados, abertos e de natureza comunitária ou territorial.

Como consequência desses diversos acontecimentos, iniciou-se um processo de revisão da legislação vigente. Em 6 de abril de 2001, o Congresso Nacional aprovou a lei 10.216 que dispõe sobre a proteção e os direitos das pessoas portadoras de transtornos mentais e redireciona o modelo assistencial em saúde mental ${ }^{11}$. Em Minas Gerais, as leis estaduais no 11.802 , de 1995 e no 12.684 de 1997 já preconizavam a extinção progressiva dos hospitais psiquiátricos e sua substituição por uma rede de serviços abertos e regionalizados ${ }^{12}$, constituindo uma proposta ainda mais incisiva de desospitalização.

Apesar das melhorias alcançadas com a instituição de serviços comunitários de atenção à saúde mental, que priorizam a manutenção do usuário em seu contexto relacional e evitam internações desnecessárias, a implantação dessa proposta de reforma na prática não se dá sem percalços. Diversos aspectos práticos da reforma 
têm sido alvo de críticas, incluindo: a centralização do modelo nos Centros de Atenção Psicossocial (CAPS) em detrimento de outras instâncias; a insuficiência da criação de serviços com funcionamento diuturno; a iniquidade de acesso nas diversas regiões do País; a judicialização das internações; e a escassez de avaliações de custo-efetividade das reformas implantadas ${ }^{13}$.

No Estado de Minas Gerais é possível perceber vastos efeitos na mudança da prática assistencial. Segundo dados da Secretaria do Estado, no início da década de 90, existiam 36 hospitais psiquiátricos perfazendo um total de 8.087 leitos no estado. Já em meados de 2011 esse número era de 2.242 leitos distribuídos em 17 hospitais. Por outro lado, o número de serviços comunitários implementados a partir da década de 90 chegou a 173 CAPS; 272 leitos em Hospitais Gerais; cerca de 300 equipes de Saúde Mental no PSF; 12 Centros de Convivência e 77 Serviços Residenciais Terapêuticos em dezembro de $2010^{14,15}$.

Belo Horizonte conta atualmente com dois hospitais psiquiátricos públicos para o atendimento de pacientes adultos em situação de crise: o Instituto Raul Soares (IRS), atualmente com 108 leitos, e o Hospital Galba Velloso (HGV), com 145 leitos operacionais. Esses hospitais constituem os únicos leitos disponíveis pelo SUS na cidade já que, desde 2002, Belo Horizonte não conta mais com hospitais psiquiátricos privados conveniados com a rede pública para novas internações ${ }^{16,17}$.

Para compreender mais detalhadamente o papel atual dessas instituições no contexto da reforma da assistência, este estudo visa descrever a evolução temporal dos atendimentos realizados nos hospitais psiquiátricos públicos de Belo Horizonte de 2002 a 2011, explorando mudanças nas características dos atendimentos e no perfil dos pacientes atendidos no período subsequente à sua implantação nacional.

\section{Material e métodos}

Este é um estudo descritivo, de séries temporais.

\section{Local do Estudo}

O estudo foi realizado nos hospitais psiquiátricos Hospital Galba Velloso (HGV) e Instituto Raul Soares (IRS) ambos localizados na cidade de Belo Horizonte (MG) e pertencentes à Fundação Hospitalar do Estado de Minas Gerais (FHEMIG). Estas duas instituições englobam todos os leitos em hospitais psiquiátricos disponíveis pelo
SUS em Belo Horizonte para atendimento aos pacientes acima de 18 anos provenientes de todo o Estado de Minas Gerais.

\section{Amostra}

Foram utilizados os registros informatizados dos atendimentos realizados nos setores da emergência (consultas e observação) e nas alas de internação, disponíveis integralmente no período de 2002 a 2011 em forma de planilha eletrônica. Como rotina administrativa desses hospitais as informações são cadastradas na chegada do paciente à instituição e são concluídos todos os campos quando de sua alta pelo funcionário do Serviço de Prontuários do Paciente (SPP) a partir dos dados do prontuário, como do sumário de alta e encaminhamento médico.

Os registros incluem as seguintes informações: nome do paciente, número do prontuário, data de nascimento, idade à admissão, sexo, endereço completo, diagnóstico principal e secundário pela CID-10, data e hora da admissão, data e hora da alta e encaminhamento do caso.

\section{Variáveis do estudo}

Foram coletados os dados relativos às características sociodemográficas da população atendida na urgência destes serviços (sexo, idade, procedência/local de residência) bem como o número de atendimentos realizados, número de pacientes atendidos, hipótese diagnóstica (diagnóstico registrado à alta) e tipo de atendimento de acordo com o tempo de permanência do paciente na instituição. Ainda quanto ao tipo de atendimento, os dados foram classificados como atendimento externo quando a permanência hospitalar foi menor que 4 horas; observação quando essa permanência foi de 4 a 24 horas e internação quando a permanência na instituição ultrapassou 24 horas. Para análise das reinternações, foram considerados os registros nos quais o paciente foi reinternado em até 365 dias da internação precedente, categorizando readmissão ocorrida em curto prazo $(<7$ dias da admissão precedente), médio prazo (7-30 dias da admissão precedente) e tardia (31-365 dias da admissão precedente). As hipóteses diagnósticas levantadas foram codificadas, para fins de registro estatístico, de acordo com a 10 a edição da Classificação Internacional de Doenças (CID-10). No presente trabalho, foi utilizado apenas o diagnóstico primário, por considerar que esse seria o principal motivo associado com a visita ao hospital. 


\section{Análise estatística}

Para a análise descritiva dos atendimentos e das internações realizadas, foram computadas proporções para as variáveis categóricas e médias e respectivos desvios-padrão para as contínuas. Em todos os casos, o alfa foi estabelecido em 5\%, bicaudado. Para a comparação das proporções por sexo dos atendimentos em 2002 e 2011 foi utilizado o teste de qui-quadrado para heterogeneidade. As análises de tendência foram realizadas mediante regressões lineares simples (e quadráticas, quando significativas), em que a variável resposta era o número anual de atendimentos ou internações (totais e por tipo) ou a proporção de atendimentos/internações por diagnóstico ou procedência e a variável preditora era o ano. Para a comparação das medianas anuais do tempo de internação foi utilizado o teste não paramétrico de Kruskal-Wallis.

\section{Aspectos Éticos}

Este projeto foi aprovado pelos Comitês de Ética em Pesquisa (COEP) da Fundação Hospitalar do Estado de Minas Gerais (FHEMIG) e da Universidade Federal de Minas Gerais (UFMG). Os dados confidenciais contidos nos bancos de dados foram mantidos sob anonimato, assegurando a privacidade dos sujeitos.

\section{Resultados}

\section{Atendimentos efetuados no período}

\section{Idade e Sexo}

Houve um ligeiro aumento na proporção de atendimentos a pacientes do sexo masculino entre 2002 e 2011 , de $57,3 \%$ para 59,6\% $(\mathrm{p}=0,026)$. A idade dos pacientes atendidos apresentou uma média de 37,9 anos em 2002 com um crescimento linear no período de 0,15 anos de idade a cada ano $\left(\mathrm{R}^{2}=0,1 \% ; \mathrm{p}<0,001\right)$.

\section{Número total de atendimentos por ano}

Em 2002, o número total de atendimentos na emergência destes hospitais foi de 18.114, caindo para 12.618 em 2011. Observou-se redução linear do número total atendimentos neste período, estatisticamente significativa, de 542 atendimentos por ano (média anual $=16.015 ; \mathrm{R}^{2}=$ $86,3 \% ; \mathrm{p}<0,001)$.

\section{Tipo de atendimento}

O número de atendimentos realizados na urgência dos dois hospitais estudados, em todo o período pesquisado, foi de 160.437 atendimentos, correspondendo a uma média de 1.337 atendimentos mensais.

Em relação ao tipo de atendimento realizado segundo o tempo de permanência hospitalar (Figura 1), observou-se redução linear significativa no número de atendimentos externos de 525 atendimentos ao ano (média anual $=9.474 ; \mathrm{R}^{2}=$ 90,5\%; p < 0,001) e no número de observações clínicas foi encontrada uma redução linear também significativa de 101 ao ano (média anual = 2.276; $\left.\mathrm{R}^{2}=65,3 \% ; \mathrm{p}=0,008\right)$. No entanto, houve um aumento linear das internações realizadas no período, de 83,8 ao ano (média anual $=4.265 ; \mathrm{R}^{2}$ $=46,7 \% ; \mathrm{p}=0,018)$.

\section{Procedência}

Dos 160.437 atendimentos de 2002 a 2011, $54,1 \%$ foram procedentes de Belo Horizonte, $34,8 \%$, da região metropolitana, $10,9 \%$, oriundos de outras cidades do Estado de Minas Gerais e 0,2\%, de outros Estados (Figura 2).

Houve uma redução estatisticamente significativa na proporção de atendimentos a procedentes da capital no ritmo de $1,0 \%$ ao ano $\left(\mathrm{R}^{2}=\right.$ $83,2 \%$; $<<0,001)$. Simultaneamente observou-se um aumento significativo de $0,9 \%$ ao ano $\left(\mathrm{R}^{2}=\right.$ $81,5 \%$; $\mathrm{p}<0,001)$ dos procedentes da outras cidades da região metropolitana de Belo Horizonte. Não houve variação estatisticamente significativa na proporção de atendimentos a pacientes procedentes de outros municípios ou estados.

\section{Diagnósticos}

Dos 160.437 atendimentos realizados em todo o período $31,2 \%$ tinham o diagnóstico de Esquizofrenia, Transtornos Esquizotípicos e Delirantes (F20-F29); 23,5\% correspondiam a Transtornos Mentais e de Comportamento Decorrentes do Uso de Álcool/Substâncias Psicoativas (F10-F19); 17,7\%, Transtornos do humor (F30-F39); 10,1\% Transtornos Neuróticos, Relacionados ao Estresse e Somatoformes (F40-F49), e 5,9\% não tinham hipótese diagnóstica registrada (Figura 3).

Entre 2002 e 2011, a proporção de atendimentos nos quais o motivo da procura ao hospital foi enquadrado dentro das categorias diagnósticas F20-F29 apresentou redução linear 
Figura 1. Número de atendimentos por tempo de permanência, Hospital Galba Velloso e Instituto Raul Soares, 2002 a 2011.

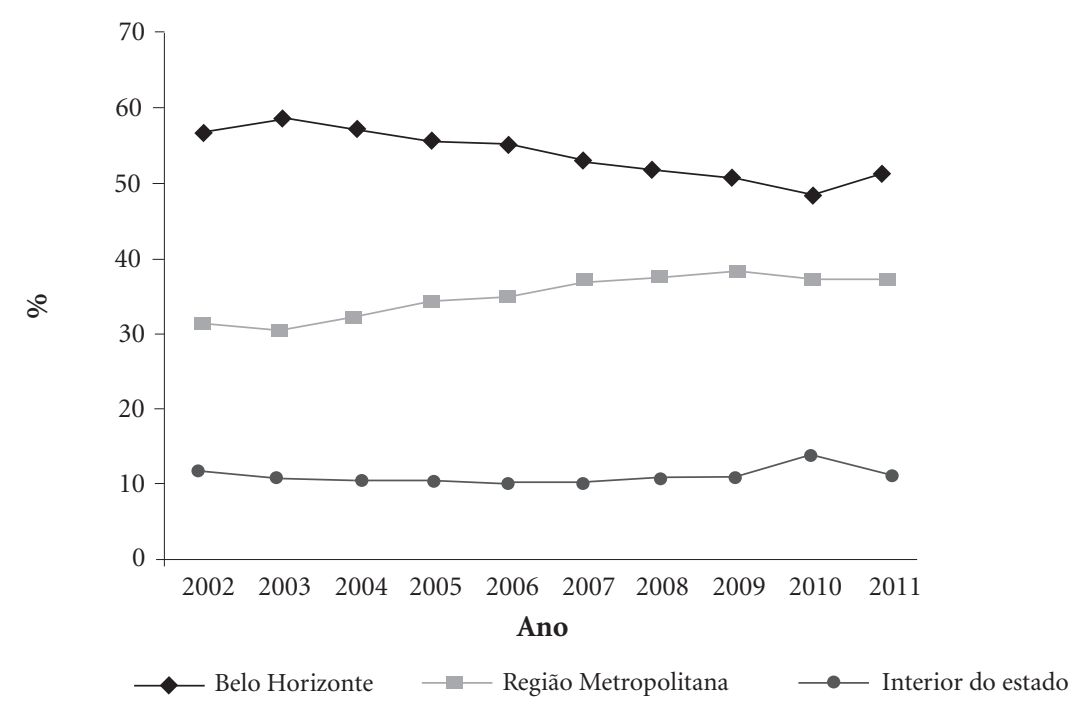

Figura 2. Proporções de atendimentos por procedência, Hospital Galba Velloso e Instituto Raul Soares, 2002 a 2011.

significativa de $0,8 \%$ a cada ano (média anual $=$ 4.993; $\left.\mathrm{R}^{2}=87,1 \% ; \mathrm{p}<0,001\right)$. A proporção de atendimentos relativos às categorias diagnósticas F10-F19 (média anual: 2.835) apresentou uma variação quadrática, com redução até 2006 e aumento acentuado de 2007 em diante $\left(R^{2}=\right.$ $86,2 \% ; \mathrm{p}=0,001)$. Atendimentos por Transtor- nos do Humor (F30-F39) apresentaram um ligeiro aumento percentual de $2,4 \%$ no período ( $\mathrm{p}=0,048)$ e os ligados a F40-F49 não tiveram alteração significativa. Podemos observar que em 2011 houve uma inversão na categoria diagnóstica mais prevalente nos atendimentos que deixou de ser F20-29 e passou a ser F10-19. 


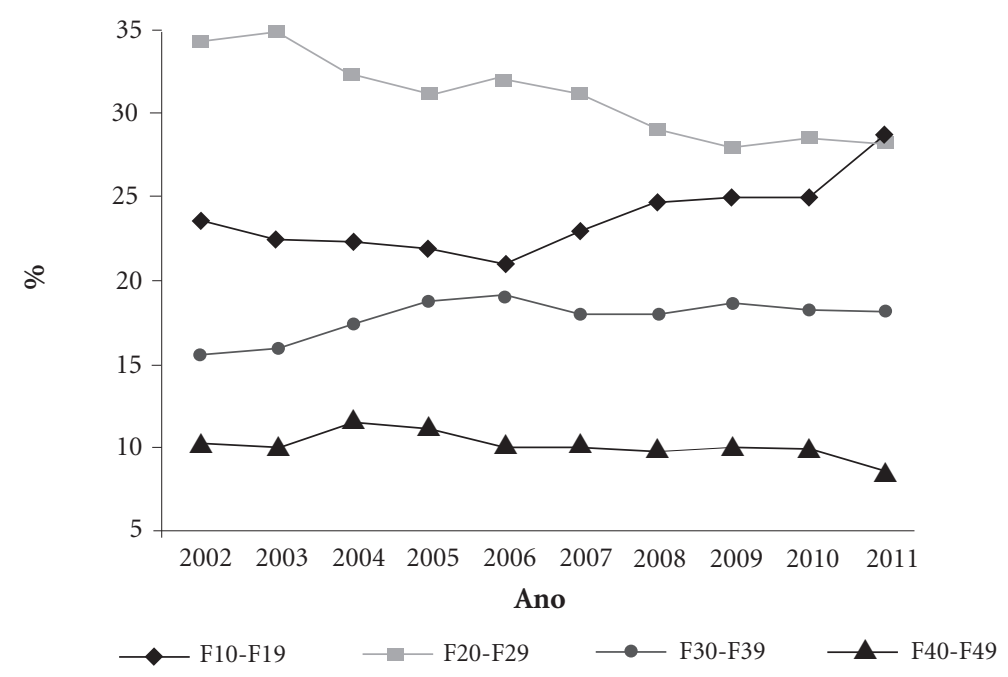

Figura 3. Proporções de atendimentos por categorias diagnósticas (CID-10), Hospital Galba Velloso e Instituto Raul Soares, 2002 a 2011.

\section{Internações realizadas no período}

\section{Idade e Sexo}

A proporção de internações de pacientes do sexo masculino entre 2002 e 2011 aumentou de $59,8 \%$ para $66,3 \%(\mathrm{p}<0,001)$. A idade dos pacientes atendidos apresentou uma média de 37,2 anos em 2002, com um crescimento linear no período de 0,12 anos de idade a cada ano $\left(\mathrm{R}^{2}=\right.$ $0,1 \% ; \mathrm{p}<0,001)$.

\section{Procedência}

Houve uma redução estatisticamente significativa na proporção de internações de procedentes da capital no ritmo de $0,6 \%$ ao ano $\left(\mathrm{R}^{2}=\right.$ $55,4 \% ; \mathrm{p}=0,008)$. Simultaneamente observou-se um aumento significativo de $1,0 \%$ ao ano $\left(\mathrm{R}^{2}=\right.$ $78,5 \%$; $<<0,001)$ dos procedentes da outras cidades da região metropolitana de Belo Horizonte. Houve também uma redução de $0,5 \%$ ao ano $\left(\mathrm{R}^{2}=62,6 \% ; \mathrm{p}=0,004\right)$ nas internações referentes a pacientes do interior de Minas Gerais entre 2002 e 2011. Não houve variação estatisticamente significativa na proporção de internações de pacientes procedentes de outros estados.

\section{Diagnósticos}

Analisando especificamente as 42.598 internações ocorridas no período, 44,2\% foram diagnosticadas como F20-29; 26,5\% como F10-F19;
17,8\% como F30-F39 e 2,8\% como F40-49. Outras categorias diagnósticas foram responsáveis por $8,7 \%$ dos diagnósticos de alta registrados e $0,1 \%$ dos registros não continham diagnóstico registrado (Figura 4 ).

Entre 2002 e 2011, a proporção de internações com diagnósticos enquadrados dentro das categorias diagnósticas F20-F29 apresentou redução linear significativa de $1,7 \%$ a cada ano $\left(\mathrm{R}^{2}\right.$ =93,0\%; $\mathrm{p}<0,001)$. A proporção de internações relativas às categorias diagnósticas F10-F19 apresentou um aumento linear de $2,0 \%$ ao ano $\left(\mathrm{R}^{2}=\right.$ $92,1 \%$; $\mathrm{p}<0,001)$. Não houve variação estatisticamente significativa na proporção de pacientes internados por outras categorias diagnósticas.

\section{Tempo de internação}

No período estudado houve mudanças significativas $(\mathrm{p}<0,001)$ nos tempos de internação medianos, passando de 12 dias em 2002 a 8 dias após 2008, mantendo-se assim estável até 2011.

\section{Reinternações}

A taxa de reinternação nos hospitais estudados, de 2002 a 2011, foi estimada em 24,6\%. Dessas reinternações, 5,3\% ocorreram em até sete dias da alta anterior; $14,6 \%$ entre 8 e 30 dias e $80,1 \%$ constituíram readmissões entre 31 e 365 dias. Não houve diferença estatística significativa nessas taxas entre 2002 e 2011. 


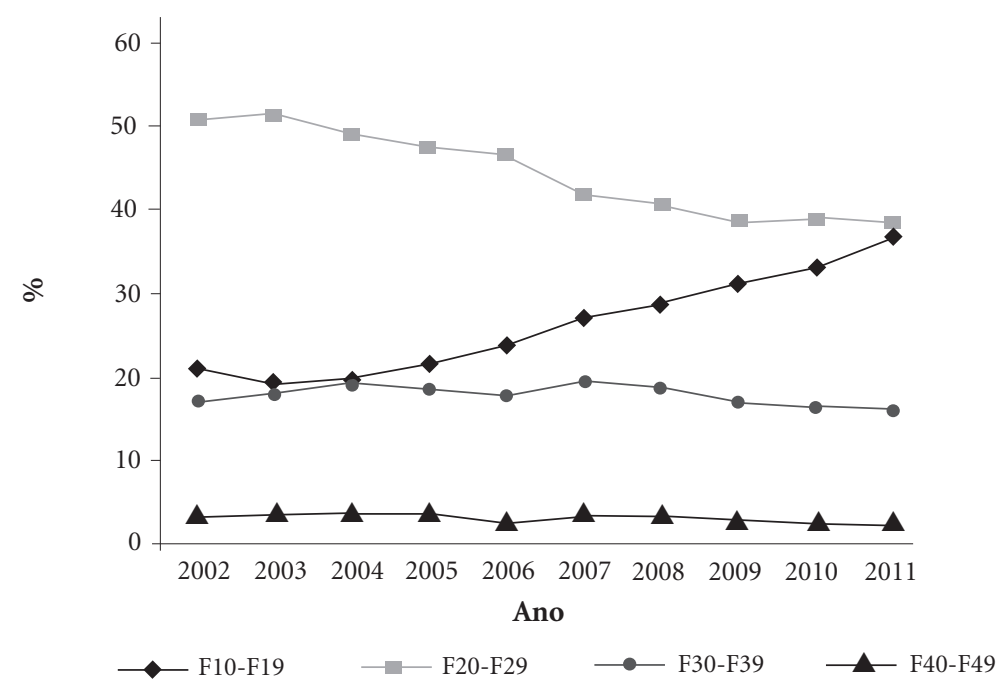

Figura 4. Proporções de internações por categorias diagnósticas (CID-10), Hospital Galba Velloso e Instituto Raul Soares, 2002 a 2011.

\section{Discussão}

A efervescência das últimas décadas, representada pelos debates e efetivas transformações no âmbito da saúde mental no Brasil, vem ocorrendo com limitado acompanhamento epidemiológico $^{18}$. Não obstante, a Organização Mundial de Saúde (OMS) preconiza que a melhoria da saúde mental supõe investimento na produção de dados concretos sobre os serviços e recursos existentes de modo que se possa definir uma estratégia de avaliação contínua de toda atividade nova ${ }^{19}$. É nesse contexto que se insere a presente pesquisa, produzindo informações relevantes para nortear as políticas e propostas de gestão pública da saúde mental.

A heterogeneidade entre as reformas na assistência conduzidas nos diversos países dificulta a comparação objetiva de estratégias ${ }^{20-22}$. Nesse contexto, a necessidade de produzir dados para a avaliação das mudanças nos serviços de saúde mental brasileiros já foi apontada por outros autores ${ }^{13,18,23}$ e constitui a essência do presente artigo.

A maior prevalência de homens nas internações psiquiátricas já foi relatada em outros traba$\operatorname{lhos}^{24-27}$ e foi consistente com os resultados deste estudo. Hipotetiza-se que os homens possam apresentar uma maior impulsividade e agressividade no curso dos transtornos psiquiátricos, $\mathrm{o}$ que justificaria um maior encaminhamento desses pacientes para o tratamento hospitalar.
Apesar de ter havido um crescimento populacional no estado de Minas Gerais de 18.343.517 habitantes em 2002 para 19.728 .252 em $2011^{28}$ houve uma diminuição importante no número de atendimentos realizados pelos hospitais psiquiátricos estaduais integrantes do SUS de Belo Horizonte (MG) nesse período. Essa evolução foi concomitante com a estruturação progressiva dos serviços de atendimento a saúde mental comunitários no estado de Minas Gerais, ocorridas após a aprovação da lei $10.261 \mathrm{em}$ 2001. De fato, Minas Gerais contava com 0,26 CAPS/100.000 habitantes em 2002 chegando a 0,69 CAPS/ 100.000 habitantes em $2011^{14}$.

Durante o período analisado houve queda significativa nos atendimentos com permanência hospitalar inferior a 4 horas e entre 4 e 24 horas que correspondem respectivamente aos atendimentos externos e observações clínicas realizados no âmbito hospitalar. Tais achados também estão de acordo com a expansão da rede comunitária de atendimento que possivelmente tem suprido uma demanda que antes se dirigia às urgências dos hospitais psiquiátricos.

Inversamente ocorreu um aumento significativo do número de internações realizadas, com diminuição de sua duração mediana e manutenção da taxa de reinternação, apesar da constatação da redução do número de leitos nos hospitais estudados de 259 em 2002 para 253 leitos em 2011. Diante desse volume elevado de interna- 
ções, pode-se hipotetizar que alguns casos, dada sua complexidade, natureza e gravidade, continuaram necessitando da assistência hospitalar, mesmo que de curta duração.

Com relação a esse aspecto, apesar dos dados institucionais demonstrarem o crescimento dos serviços substitutivos e a queda nos leitos psiquiátricos ${ }^{14}$, outros estudos ${ }^{24,29-34}$ já constataram o tímido crescimento dos serviços comunitários que possuem funcionamento 24 horas, bem como o daqueles voltados aos cuidados de populações especificas, como os CAPSi (destinados ao atendimento de crianças e adolescentes) e os CAPSad (destinados ao atendimento de pacientes portadores de transtornos secundários ao uso ou abuso de álcool ou outras drogas). Em 2011, do total de 1742 CAPS, existiam em todo território brasileiro somente 63 CAPS 24 horas, 149 CAPSi e 272 CAPSad $^{14}$.

A política nacional de saúde mental preconiza que as internações psiquiátricas hospitalares, quando necessárias, se realizem em hospitais gerais. Entretanto, analisando os números oficiais, até 2011, foram criados somente 3.910 leitos nesses hospitais em todo o país ${ }^{14}$. Em MG foram criados, até 2011, 272 leitos em hospitais gerais. Por outro lado, existiam, em 2011, 17 hospitais psiquiátricos em todo o estado com 2.242 leitos destinados ao SUS ${ }^{15}$.

Com relação à morbidade prevalente, estudos descritivos realizados em hospitais psiquiátricos conveniados ao SUS em outros estados (RJ, BA e MS) foram consistentes ao encontrar uma maior prevalência de Esquizofrenia, Transtornos Esquizotípicos e Delirantes nos atendimentos ${ }^{25,26,35}$.

Um dos achados mais marcantes do presente estudo é a tendência de inversão nas morbidades mais frequentes nesses hospitais, com os transtornos ligados a abuso e dependência de substâncias ultrapassando o número de atendimentos por transtornos psicóticos no ano de 2011. É improvável que este fenômeno se associe diretamente a variações de prevalências na comunidade ${ }^{36}$. Ao contrário, é razoável supor que o fenômeno esteja associado com a marcante diferença na efetividade dos tratamentos para as psicoses e para as dependências e, também, à dificuldade de acesso aos tratamentos especializados para essas últimas.

Analisando detalhadamente a rede de atenção comunitária de Minas Gerais notamos uma desproporção nos serviços ofertados a pacientes dependentes químicos. Em 2011, Minas Gerais possuía somente 21 CAPS destinados a estes pacientes (CAPSad), em contraste com 130 CAPS e 10 CAPS 24 horas destinados a pacientes porta- dores de transtorno mental que não sejam causados pelo uso de álcool ou outras drogas ${ }^{15}$.

Em Belo Horizonte, existiam, em 2011, dois serviços destinados a usuários de álcool e outras drogas pelo SUS: um CAPSad municipal e o Centro Mineiro de Toxicomania (CMT), que segue as mesmas diretrizes de funcionamento de um CAPS, porém é administrado pela FHEMIG. No censo de 2010, o IBGE estimou a população residente de Belo Horizonte em 2.375.151 $1^{28}$, levando-se em consideração o I Levantamento Domiciliar sobre o Uso de Drogas Psicotrópicas no Brasil, $12,7 \%$ da população da região Sudeste preenche critérios para dependência de álcool enquanto $2,7 \%$ desta população é dependente de drogas ilícitas ${ }^{36}$. De acordo com essas estimativas, haveria 308.770 dependentes químicos em Belo Horizonte contando com somente dois serviços comunitários especializados, sendo que em nenhum deles o funcionamento era realizado nas 24 horas. Desta forma pode-se hipotetizar que esta clientela demande atendimento nos hospitais psiquiátricos existentes, por carência de serviços comunitários especializados. Esse resultado ainda pode indicar não só uma maior fragilidade da rede de saúde mental em relação aos usuários de álcool e drogas, mas também refletir a dificuldade em se criar leitos para esses pacientes em hospitais gerais.

Os pacientes procedentes de Belo Horizonte constituíram a maior parte da clientela atendida durante todo o período. Apesar do crescimento populacional de $4,4 \%{ }^{28}$ apresentado entre 2002 e 2011 para a capital mineira, observou-se uma diminuição expressiva na proporção de atendimentos realizados a pacientes deste município ao longo do período analisado. Em contrapartida, houve um aumento proporcional dos atendimentos relativos à população da região metropolitana mesmo quando as análises foram ajustadas levando em consideração o crescimento populacional de $8,6 \%{ }^{28}$ apresentado no período para os municípios integrantes dessa região.

Essa queda apresentada nos atendimentos hospitalares realizados a pacientes provenientes da capital pode ser correlacionada temporalmente com a estruturação dos serviços com atendimento 24 horas em Belo Horizonte. Os CAPS deste município começaram a ser implantados em 1993, contando em 2002 com sete serviços em funcionamento. No que diz respeito ao atendimento das urgências/emergências psiquiátricas, o modelo adotado em 2001 pela Secretaria Municipal de Saúde foi oferecer dois CAPS 24 horas, com 6 leitos-noite em cada serviço. Em 
2006, buscando uma melhor integração e construção de uma rede de atendimento às situações de crise, foi implantada a "Hospitalidade Noturna” em todos os sete CAPS e criado o Serviço de Urgência Psiquiátrica (SUP), localizado na região central da cidade, com seis leitos e funcionando das 19 às 7 horas $^{12}$.

$\mathrm{O}$ aumento relativo observado na proporção de pacientes provenientes dos outros municípios da região metropolitana está associada, paradoxalmente, à existência de uma rede de atenção extra-hospitalar com maior razão por habitante ${ }^{37}$. Apesar dessa aparente contradição, deve-se considerar que a implantação de serviços comunitários por si só não abrange a complexidade que a atenção em saúde mental requer. Outros fatores que não foram diretamente analisados neste trabalho merecem ser estudados em estudos posteriores para melhor esclarecimento desse ponto como, por exemplo, o modo de funcionamento dos CAPS em municípios de pequeno e médio porte, os fatores socioeconômicos inerentes às regiões metropolitanas e as particularidades de cada rede analisada.

As modificações introduzidas pelo movimento de reforma psiquiátrica no modo de trabalho com a saúde mental trazem importantes reflexões. O fenômeno de desospitalização dos pacientes psiquiátricos ocorreu em diversos países, mesmo que de diferentes maneiras e meios. Essa população, previamente confinada em manicômios, agora vive entre a sociedade. Gradualmente, tem-se aceitado que o tratamento psiquiátrico pode ser fornecido dentro da comunidade, ao invés de em grandes instituições. Essa nova abordagem traz a ideia de que os serviços comunitários podem responder às necessidades dos indivíduos de uma maneira diferente do que é feito no hospital psiquiátrico. O início do tratamento psicofarmacológico trouxe importantes inovações: a introdução dos neurolépticos em 1952 possibilitou a continuidade do tratamento fora do âmbito hospitalar, além de atuar sobre os comportamentos dos doentes mentais que geralmente ocasionavam sua internação. A difusão da consciência sanitária estimulou os pacientes, familiares, profissionais da área e políticos a exigirem melhores condições de vida e de cuidados para os pacientes psiquiátricos ${ }^{38}$.

A dilatação da psiquiatria, com ampliação de seu campo para o círculo social, incentivou o redimensionamento do conhecimento e das práticas que cercam a loucura. Entretanto, essa expansão inclui diversos desafios e contradições para além de suas aspirações para uma transformação paradigmática. Essa reinvenção de teorias e práticas demanda uma avaliação crítica da nossa realidade de cuidados que ultrapassa a desconstrução de aparatos antigos e práticas relativas ao paciente e sua loucura, mas engloba a complementariedade ao contrário do absolutismo ${ }^{38}$.

Dessa forma, a manutenção do hospital psiquiátrico no circuito de atendimento da saúde mental nos leva à análise das dificuldades enfrentadas na efetivação da rede comunitária de saúde mental como substitutiva de fato.

No caso particular de Belo Horizonte, alguns estudos analisaram esses aspectos. Silva ${ }^{39}$, em pesquisa qualitativa realizada nos CAPS de Belo Horizonte e Betim, questiona esse caráter substitutivo no funcionamento atual dos CAPS. Segundo esse trabalho, os CAPS encontram-se "psiquiatrizados e medicalizados, na sequência, ambulatorizados, desvitalizados e carentes de uma reconfiguração”. Através do estudo de caso realizado, ele infere que "talvez a questão esteja na inflexão de processos ideológicos mais acirrados, na desvalorização dos saberes investidos e na falta de diálogo entre gestores e trabalhadores sobre a atividade dentro de um projeto comum".

Dessa forma, este estudo questiona se haveria uma contradição no processo da reforma psiquiátrica, uma distância entre o ideal prescrito e o real da prática clínica nos serviços substitutivos que levaria a uma reprodução de práticas criticadas pelo movimento reformista. Nesse sentido, Anaya ${ }^{40}$, também em pesquisa acerca dos CAPS de Belo Horizonte, pontua que esses serviços esforçam-se cotidianamente na busca de se tornarem substitutivos de fato. Contudo, tem-se deparado com diversos impasses tais como: dificuldades de articulação na rede de saúde mental do qual são referência, atendimento de demanda superior à que deveriam atender, desvio da maioria dos trabalhadores para o plantão, reduzindo a flexibilização do trabalho em atividades criativas e não reprodutoras do modelo tradicional psiquiátrico de assistência, dentre outros.

Além desses pontos, outros autores ${ }^{29-34}$ sinalizam dificuldades atuais para a implementação da rede substitutiva: a centralização do modelo nos CAPS com pouco incentivo aos outros componentes da rede de cuidados; as dificuldades para inserção da atenção básica na rede de cuidados em saúde mental; a insuficiência da criação de serviços com funcionamento 24 horas; as disparidades existentes no acesso da população ao tratamento nas diversas regiões do Estado; a judicialização das internações e a necessidade de avaliação continua do modelo de assistência. Além 
dessas questões, observa-se a escassez de recursos comunitários para atendimento aos dependentes químicos que constituem, atualmente, a principal demanda de atendimento dos hospitais psiquiátricos estudados.

De fato, apesar do crescente aprimoramento apresentado pela rede de saúde substitutiva de Belo Horizonte, notamos que, em 2011, período final deste estudo, os pacientes dessa capital ainda são os mais prevalentes no atendimento dos hospitais analisados. Esse dado fornece subsídios para futuras pesquisas que possam analisar o motivo pelo qual, no momento atual, os pacientes pertencentes a áreas providas de CAPS continuam buscando atendimento no hospital psiquiátrico.

Apesar da ideia corrente de que manter o hospital psiquiátrico no imaginário coletivo, como um abrigo seguro para as situações críticas, constituiria um retrocesso no processo da reforma psiquiátrica ${ }^{30}$, deve-se considerar que inexiste sistema de saúde mental que possa funcionar sem garantia de atendimento à crise ${ }^{29}$. A redução de leitos em hospitais psiquiátricos deve ser acompanhada da implantação de uma rede comunitária que atenda às necessidades dos usuários. Quanto mais abrangente e eficiente for este sistema menor a necessidade de admissão hospitalar ${ }^{32}$.

\section{Conclusão}

Pôde-se observar uma alteração no perfil de atendimento realizado pelos hospitais psiquiátricos públicos de Belo Horizonte de 2002 a 2011. Ocorreu redução dos atendimentos externos, aumento do número de internações (embora de menor duração), redução relativa dos atendimentos de oriundos da própria capital, e surgimento de predominância de diagnósticos de transtornos ligados ao uso de álcool e drogas.

Devido à natureza descritiva do presente estudo, não se pode afirmar que as mudanças implementadas pela reforma na assistência foram as responsáveis por essa modificação. Entretanto, pode-se hipotetizar que a instituição de serviços comunitários para atendimento à saúde mental tenha retirado o hospital do foco da assistência à essa população.

Atualmente o hospital psiquiátrico não constitui a primeira e muito menos a única instância de tratamento, todavia constatou-se que essas instituições ainda fazem parte do circuito de atendimento da rede de saúde mental de Minas Gerais, principalmente no que se refere à internação breve dos pacientes. Dessa forma, deve-se considerar que, até o momento, pacientes com um perfil específico ainda utilizam a assistência hospitalar, ainda que de curta duração.

As perspectivas da reforma psiquiátrica não devem se esgotar no combate ao hospital como local de tratamento. É necessário considerar a complexidade e o polimorfismo das experiências de sofrimento mental para que se possa fornecer redes de atendimento que contemplem as singularidades de cada sujeito/paciente/usuário nesse percurso.

\section{Colaboradores}

VAA Coelho trabalhou na concepção, delineamento e interpretação dos dados, redação do artigo e aprovação da versão final a ser publicada; FM Volpe no delineamento e interpretação dos dados, revisão crítica do artigo e aprovação da versão final a ser publicada; SSL Diniz e EM Silva na análise e interpretação dos dados, revisão crítica do artigo e aprovação da versão final a ser publicada; CF Cunha na concepção, delineamento e interpretação dos dados, revisão crítica do artigo e aprovação da versão final a ser publicada. 


\section{Referências}

1. Silveira LC, Braga VAB. Acerca do conceito de loucura e seus reflexos na assistência de saúde mental. Rev. Latino -Am. Enfermagem 2005; 13(4):591-595.

2. Foucault M. O nascimento do asilo. In: Foucault M. História da loucura na idade clássica. $6^{\mathrm{a}}$ Ed. São Paulo: Perspectiva; 2007. p. 459-504

3. Bercherie P. Os Fundamentos da Clínica: história e estrutura do saber psiquiátrico. Rio de Janeiro: Jorge Zahar; 1989.

4. Amarante P. Saúde Mental e atenção psicossocial. Rio de Janeiro: Editora Fiocruz; 2007.

5. Desviat M. A reforma psiquiátrica. Rio de Janeiro: Editora Fiocruz; 1999.

6. Alves CFO, Ribas VR, Alves EVR, Viana MT, Ribas RMG, Júnior LPM, Martins HAL, Lima MDC, Sougey EB, Castro RM. Uma breve história da reforma psiquiátrica. Neurobiologia 2009; 72(1):85-96.

7. Amarante P. Novos sujeitos, novos direitos: o debate em torno da reforma psiquiátrica. Cad Saude Publica 1995; 11(3):491-494.

8. Tenório F. Psychiatry reform in Brazil from the 1980's to present days: its history and concepts. Hist. cienc. saude-Manguinhos 2002; 9(1):25-59.

9. Goulart MSB. A construção da mudança nas instituições sociais: a Reforma Psiquiátrica. Pesquisas e práticas psicossociais 2006; 1(1):01-19.

10. Goulart MSB. O movimento antimanicomial italiano e suas conexões com o Brasil. In: Goulart MSB, organizador. As raizes italianas do movimento antimanicomial. São Paulo: Casa do Psicólogo; 2007. p. 23-34.

11. Brasil. Lei n. 10.216, de 6 de abril de 2001. Dispõe sobre a proteção e os direitos das pessoas portadoras de transtornos mentais e redireciona o modelo assistencial em saúde mental, que são assegurados sem discriminação quanto à raça, cor, sexo, orientação sexual, religião, opção política, nacionalidade, idade, família, recursos econômicos e ao grau de gravidade ou tempo de evolução de seu transtorno, ou qualquer outra. Diário Oficial da União 2001; 6 Abr.

12. Souza PJC. Resposta à Crise: A experiência de Belo Horizonte. Política de Saúde Mental de Belo Horizonte: O Cotidiano de uma Utopia [Internet]. 2008 [acessado 2012 ago 23]. 1a Ed. 111-29. Disponível em: http://xa.yimg. com/kq/groups/22370896/1330363327/name/Servico_ de_Urgencia_Psiquiatrica\%5B1\%5D.pdf

13. Associação Brasileira de Psiquiatria (ABP). Proposta de Diretrizes para um Modelo de Assistência Integral em Saúde Mental no Brasil. Brasília: ABP; 2006.

14. Brasil. Ministério da Saúde (MS). Saúde Mental em Dados [Internet]. Versão 10. Brasília: Ministério da Saúde do Brasil; 2012. [acessado 2012 ago 20] Disponível em: www.saude.gov.br/bvs/saudemental

15. Souza ME. Atenção em Saúde Mental. Belo Horizonte: Secretaria de Estado de Saúde de Minas Gerais; 2006.

16. Brasil. Ministério da Saúde (MS). Saúde Mental em Dados. [Internet]. Versão 7. Brasília: Ministério da Saúde do Brasil; 2010. [acessado $2011 \mathrm{dez} 10$ ]. Disponível em: www.saude.gov.br/bvs/saudemental

17. Volpe FM, Silva EM, Carmo LS, Santos TN. Clinical and demographical profile of patients admitted to a psychiatric emergency service of Belo Horizonte, Brazil, 2002-2007. J. Bras. Psiquiatr. 2010; 59(3):203-209.
18. Onocko-Campos RT, Furtado JP. Entre a saúde coletiva e a saúde mental: um instrumental metodológico para avaliação da rede de Centros de Atenção Psicossocial (CAPS) do Sistema Único de Saúde. Cad Saude Publica 2006; 22(5):1053-1062.

19. Organização Mundial de Saúde (OMS). Relatório sobre a Saúde no Mundo 2001: Saúde Mental: nova concepção, nova esperança. Geneva: OMS; 2001.

20. Desviat M. International overview of psychiatric reform. Cien Saude Colet 2011; 16(12):4615-4622.

21. Becker T, Kilian R. Psychiatric services for people with severe mental illness across Western Europe: what can be generalized from current knowledge about differences in provision, costs and outcomes of mental health care? Acta Psychiatr Scand Suppl 2006; (429):9-16.

22. Livingston JD, Nicholls T, Brink J. The Impact of Realigning a Tertiary Psychiatric Hospital in British Columbia on Other Institutional Sectors. Psych Serv 2011; 62(2):200-205.

23. Gentil V. Projeto Delgado, Substitutivo Rocha ou uma lei melhor? Rev. Bras. Psiquiatr 2001; 23(1):1-2.

24. Barros REM, Tung TC, Mari JJ. Psychiatric emergency services and their relationships with mental health network in Brazil. Rev. Bras. Psiquiatr. 2010; 32(Supl. 2):71-77.

25. Silva JPL, Coutinho ESF, Amarante PD. Perfil demográfico e sócio-econômico da população de internos dos hospitais psiquiátricos da cidade do Rio de Janeiro. Cad Saude Publica 1999; 15(3):505-511.

26. Souza JC, Souza N, Magna LA. Tempo médio de hospitalização e categorias diagnósticas em hospital psiquiátrico. J. Bras. Psiquiatr 2008; 57(2):112-116.

27. Gomes MPC, Couto MCV, Pepe VLE, Almeida LM, Delgado PGG, Coutinho ESF. Censo dos pacientes internados em uma instituição asilar no Estado do Rio de Janeiro: dados preliminares. Cad Saude Publica 2002; 18(6):1803-1807.

28. Brasil. Departamento de Informática do Sistema Único de Saúde (Datasus). [Internet]. Brasília. c1991. [acessado 2013 jan 10]. Disponível em: http://tabnet.datasus. gov.br/cgi/tabcgi.exe?ibge/cnv/popmg.def

29. Lobosque AM. Debatendo alguns desafios da reforma psiquiátrica brasileira. Cien Saude Colet 2011; 16(12): 4590-4602.

30. Pitta AMF. An assessment of Brazilian psychiatric reform: institutions, actors and policies. Cien Saude Colet 2011; 16(12):4579-4589.

31. Levav I. Extender la reforma por medio de nuevas acciones de salud mental. Cien Saude Colet 2011; 16(12):4590-4602.

32. Mari JJ. Um balanço da reforma psiquiátrica brasileira. Cien Saude Colet 2011; 16(12):4590-4602.

33. Junior BB. É preciso repensar o horizonte da reforma psiquiátrica. Cien Saude Colet 2011; 16(12):4598-4600.

34. Costa NR, Siqueira SV, UhrI D, Silva PF, Molinaro AA. Reforma psiquiátrica, federalismo e descentralização da saúde pública no Brasil. Cien Saude Colet 2011; 16(12):4603-4614.

35. Rabelo AR, Cardoso E, Melo A. Características sociodemográficas da população psiquiátrica internada nos hospitais do Sistema Único de Saúde do Estado da Bahia. Rev. Baiana Saúde Pública 2005; 29(1):43-56. 
36. Carlini EA, Galduróz JCF, Noto AR, Nappo SA. I Levantamento domiciliar sobre o uso de drogas psicotrópicas no Brasil: estudo envolvendo as 107 maiores cidades do país. Rev. Latino-Am. Enfermagem 2005; 13(N. Esp.):888-895.

37. Brandão PF, Mol MS, Lages CS, Siqueira MG, Francisco EL, Silva EM, Volpe FM. Avaliação epidemiológica e georeferenciada da assistência em saúde mental do Hospital Galba Velloso, 2002 a 2009. Rev. Méd. Minas Gerais 2012; 22(Supl. 2):115.

38. Pinho LB, Kantorski LP. Psychiatric care in the Brazilian context. Cien Saude Colet 2011; 16(4):2107-2114.

39. Silva ER. A atividade de trabalho do psiquiatra no CAPS - Centro de Atenção Psicossocial: pois é José... [dissertação]. Belo Horizonte: Universidade Federal de Minas Gerais; 2010.

40. Anaya F. Reflexão sobre o Conceito de Serviço Substitutivo em Saúde Mental: a Contribuição do CERSAM de Belo Horizonte-MG [dissertação]. Rio de Janeiro: Escola Nacional de Saúde Pública; 2004.

Artigo apresentado em 16/07/2013

Aprovado em 15/11/2013

Versão final apresentada em 23/11/2013 